\title{
Optimizing the prescription isodose level in stereotactic volumetric-modulated arc radiotherapy of lung lesions as a potential for dose de-escalation
}

\author{
Mark Chan ${ }^{1,2}$, Matthew Wong ${ }^{3}$, Ronnie Leung ${ }^{3}$, Steven Cheung $^{3}$ and Oliver Blanck ${ }^{1,4^{*}}$
}

\begin{abstract}
Background: To derive and exploit the optimal prescription isodose level (PIL) in inverse optimization of volumetric modulated arc radiotherapy (VMAT) as a potential approach to dose de-escalation in stereotactic body radiotherapy for non-small cell lung carcinomas (NSCLC).

Methods: For ten patients, inverse Monte Carlo dose optimization was performed to cover 95\% PTV by varying prescription isodose lines (PIL) at 60 to $80 \%$ and reference $85 \%$. Subsequently, these were re-normalized to the median gross tumor volume dose (GTV-based prescription) to assess the impacts of PTV and normal tissue dose reduction.

Results: With PTV-based prescription, GTV mean dose was much higher with the optimized PIL at $60 \%$ with significant reduction of normal lung receiving 30 to $10 \mathrm{~Gy}\left(V_{30-10 G y}\right)$, and observable but insignificant dose reduction to spinal cord, esophagus, ribs, and others compared with $85 \%$ PIL. Mean doses to the normal lung between PTV and GTV was higher with 60-70\% PIL than 85\%. The dose gradient index was $5.0 \pm 1.1$ and $6.1 \pm 1.4$ for 60 and $85 \%$ PIL $(p<0.05)$, respectively. Compared with the reference $85 \%$ PIL plan using PTV-base prescription, significant decreases of all normal tissue doses were observed with $60 \%$ and $70 \%$ PIL by GTV-based prescription. Yet, the resulting biological effective (BED) mean doses of PTV remain sufficiently high, ranging 104.2 to $116.9 \mathrm{~Gy} \alpha / \beta=10$.

Conclusions: Optimizing the PIL with VMAT has notable advantage of improving the dosimetric quality of lung SBRT and offers the potential of dose de-escalation for surrounding tissues while increasing the GTV dose simultaneously. The clinical implication of re-normalizing plans from PTV-prescription at $60-70 \%$ to the GTV median dose requires further investigations.
\end{abstract}

\section{Background}

Optimization of stereotactic body radiotherapy (SBRT) plan quality is crucial to minimize normal tissue dose and hence toxicities for inoperable early stage nonsmall-cell lung cancer (NSCLC). In lung SBRT small photon fields are widely used, which are known to introduce significant lateral electronic disequilibrium (LED) in heterogeneous tissue caused by out-scattering electrons not being compensated by in-scattering

\footnotetext{
* Correspondence: blanck@saphir-rc.com

${ }^{1}$ University Medical Center Schleswig-Holstein, Campus Kiel, Department for Radiation Oncology, Arnold-Heller-Straße 3, Haus 50, Karl-LennertKrebscentrum Nord, 24105 Kiel, Germany

${ }^{4}$ Saphir Radiosurgery Center Northern Germany, Güstrow, Germany Full list of author information is available at the end of the article
}

electrons [1]. Using the LED phenomenon for optimizing lung SBRT (LED-SBRT), which is based on the differential reductions of lung and tumor doses caused by the LED in order to steepen the dose gradients and thereby increasing the dose within the tumor while reducing the dose in the normal lung, has been recently proposed by Disher et al. [1]. However, in order for LED-SBRT to be robust against dosimetric errors, Monte Carlo (MC) simulation techniques [2] must be employed to accurately model the particle transport.

Besides direct MC simulation, the LED phenomenon was also investigated implicitly through the relationship with the prescription isodose line (PIL) by comparing the dose results from type-A pencil beam (PB), which cannot model the LED, and type-B MC based dose 
calculations [3]. Using multiple dynamic conformal arcs (DCA), Oku et al. showed a clear dependence of the PIL on the dosimetric plan quality [4]. They found higher lung dose and lower dose conformity at 50\% downwards to $20 \%$ PIL after the optimal improvement at $60 \%$ PIL with respect to the reference of $80 \%$ PIL. Similar results of optimal PIL at $\sim 60-70 \%$ and much lower at $\sim 40-50 \%$ were found for forward planned linac-based and inverse planned roboticbased SBRT lung treatments, respectively [5].

The clinical implication of lowering the PIL to $60 \%$ vs. $80 \%$ PIL in 5-fraction lung SBRT was initially studied by Takeda et al., also using DCA techniques [6]. After 6 months post-SBRT, they found no local recurrences and only a limited number of incidents of radiation pneumonitis $\geq$ Grade 2 ( 1 out of 15 patients) with lower PIL. Furthermore, Guckenberger et al. found in their retrospective large-scale multi-center analysis with low PIL $(<80 \%)$ a significant higher freedom from local progression as compared to higher PIL ( $86.8 \%$ vs. $69.1 \%$, $p=0.005$ ), again with lower toxicity for lower PIL [7]. Further evidence of the clinical effects of varying PIL was supported by a number of recent published series from European and Japanese SBRT working groups that demonstrated the iso-effectiveness of SBRT treatments between those prescribing the biological effective dose $(\mathrm{BED})>100 \mathrm{~Gy}_{10}(\alpha / \beta=10$ Gy for NSCLC) to the isocenter, to the PTV periphery with $95 \%$ coverage $\left(D_{95 \%}\right)$, and to the GTV mean or median dose [8-13].

Despite the similar local control rates reported based on different prescription concepts, the inconsistency of the conventional PTV prescription concept has been well acknowledged in the latest published International Commission on Radiation Units and Measurements (ICRU) report 91 [14], pointing at increased variability of the internal GTV dose for lung SBRT. Although the report hinted at a possible solution by using a GTVbased prescription and a few other studies [15-17] coherently showed more consistent GTV dose when renormalizing or prescribing the treatment dose to the GTV mean or median dose or $D_{99 \%}$, the implications of such GTV-based re-normalization/re-prescription with respect to the PIL in the conventional PTV prescription concept has never been studied.

In this work, we focused on the technical feasibility of enhancing the dosimetric quality of inverse VMAT optimization by assessing the optimal PIL and with that the potential for dose de-escalation. Further on the hypothesis that the potential dependence of plan quality on the PIL is related to the LED, retrospective PB recalculations were performed for all direct $\mathrm{MC}$-optimized VMAT plans to assess the relationship of the PIL with the dosimetric changes. Ultimately, we tried to investigate the GTV-based re-normalization / re-prescription concept with respect to the PIL for VMAT LED-SBRT.

\section{Methods}

\section{Patient selections and 4DCT imaging}

This retrospective dosimetric study, approved by the local clinical and research ethics committee, included ten patients with primary NSCLC. The tumor size ranged from 11.7 to $82.6 \mathrm{~cm}^{3}$. Each patient had a fourdimensional computed tomography (4DCT) scan that was sorted into ten 3DCT image datasets with equal time share. Each 3DCT had a slice thickness of $2 \mathrm{~mm}$ in the axial direction. We determined the mid-ventilation (MidV) phase from the 4DCT series for treatment planning as described previously [18].

\section{Treatment planning and dose prescription}

The GTV was contoured based on standard international guidelines and was expanded according to the mid-ventilation PTV concept [19]. Treatment prescription was 54 Gy in 3 fractions regardless of tumor location and size.

Dose constraints for the target and other OARs were defined following the guidelines in the ROSEL [20] and RTOG 0236 [21, 22] trials. It was demanded that dose to 95\% of the PTV (PTV D $95 \%$ ) and 98\% of the GTV (GTV $\left.D_{98 \%}\right)$ must at least receive the prescription dose. Also the dose to $98 \%$ of PTV (PTV $D_{98 \%}$ ) had to exceed $90 \%$ of the prescription dose. Furthermore, we limited the maximum dose in the PTV to be within $167 \%$ of the prescription dose despite the fact that the optimal dose gradient that may occur at as low as 30\% PIL [1] because very limited clinical trials reporting safety and toxicities with $\mathrm{PIL}<60 \%$ were available. Dose limits of chest wall and rib may be exceeded in case of large overlap with the PTV, as suggested in the RTOG 0915 trial [23].

\section{VMAT-based PIL-SBRT optimization}

For all patients, volumetric-modulated arc radiotherapy (VMAT) optimizations of lung SBRT plans were performed using the Monaco treatment planning system (TPS, v.5.0, Elekta, Sweden) for the Elekta Agility ${ }^{\text {min }}$ linear accelerator (linac) equipped with a multileaf collimator (MLC) with 160 leaf pairs of $5 \mathrm{~mm}$ width. The VMAT plans consisted of two either full or partial 6 Mega-voltage (MV) coplanar arcs, depending on the location of the tumor to avoid direct beam entrance to the contralateral lung and other central organs at risk (OAR).

For each patient, a reference plan at 85\% PIL was generated while keeping the dose constraints of the OARs as low as reasonably achievable (ALARA). Unlike the strategy with DCA, where the beam penumbra margin is manually adjusted to achieve varying levels of PIL, the MLC shapes in VMAT are completely controlled by the optimizer in achieving the dose-volume histogram (DVH) objectives. To implicitly manipulate the PIL in the inverse optimization process, we varied the maximum dose to the 
PTV and the GTV together with demanding higher minimum dose or DVH constraints to the GTV to achieve a lower or higher PIL. Dose constraints of all other OAR were kept unchanged in all inverse optimized plans for each patient to minimize the planner-related bias on the final plan quality as much as possible.

Using these optimization strategies, four plans were generated, with the reference corresponding to $85 \%$ PIL, and the other three corresponding to $80 \%, 70 \%$ and $60 \%$ PILs. All VMAT plans were directly optimized by Monte Carlo (MC) simulation technique [24] and the final dose distributions were calculated to dose to medium in medium $\left(D_{m, m}\right)$ using $2 \mathrm{~mm}$ dose grid and $0.5 \%$ relative statistical uncertainty.

\section{Plan evaluation and statistical analysis}

Dosimetric parameters of the reference plan and the PIL-optimized plans as defined in ROSEL [20] and RTOG 0236 [21, 22] trial protocols were compared, which included the normalized volume received at least 5 to 30 Gy $\left(V_{5-30 G y}\right)$ by the normal lungs, the absolute volume received at least 30 Gy and $100 \%$ of the prescription dose by the chest wall, dose to $1 \%\left(D_{1 \%}\right)$ of the cord, trachea, bronchus, esophagus and heart. The nearminimum dose $D_{98 \%}$ to PTV and GTV mean dose ( $\left.D_{\text {mean }}\right)$ were also included. The target dose conformity was assessed using the Paddick's conformity index [25]. The dose gradient was evaluated by the ratio of $50 \%$ prescription isodose volume to the PTV $\left(R_{50 \%}\right)$, and its constraint was adapted according to Xiao et al. [22] based on their retrospective re-evaluation of the dosimetric effects of heterogeneity corrections for the case submissions in the RTOG 0236 trial [21].

Further on the hypothesis that the potential dependence of plan quality on the PIL is related to the LED, all the direct $\mathrm{MC}$-optimized plans were re-calculated by a PB dose engine developed by Jelen et al. [26] The resulting difference was evaluated based on mean dose and $D_{2 \%}$ of the PTV border (i.e., PTV minus GTV) because it coincided with the low density tissue and the field edge where the LED was likely most severe.

Following the GTV-based prescription approach described by Bibault et al. [8] and Muira et al. [16], all original PTV-based prescription plans of varying PILs were re-normalized such that the GTV $D_{50 \%}$ equals 54 Gy. The resultant GTV-prescribed plans at the optimal $60 \%$ and $70 \%$ PILs were compared to the reference PTV-based prescription plans at $85 \%$ PIL to study the implications of varying PILs in different prescription concepts.

Statistical comparisons of various plan quality parameters between plans of different PILs were performed by Freidman's test. When statistical significance was found, further post-hoc test by the default Tukey's honest significant difference criterion was performed using the Matlab statistics toolbox (MathWorks, Natick, MA). Further, Wilcoxon's test was performed to assess the significance of normal tissue dose reductions achieved with GTV-based prescription compared with the reference 85\% PIL plan with PTV-based prescription. Test results were considered statically significant at $p<0.05$.

\section{Results \\ VMAT-based PIL-SBRT optimization}

GTV mean doses $\left(D_{\text {mean }}\right)$ increased from $85 \%$ PIL by $6.1 \pm 2.2 \%$ (mean \pm 1 standard deviation (SD)), $19.2 \pm$ $3.3 \%$, and $35.0 \pm 5.5 \%$ with decreasing PILs to $80 \%, 70 \%$ and $60 \%$, respectively $(p<0.01)$. The mean near-minimum dose $D_{98 \%}$ of PTV showed an observable but insignificant descending trend with decreasing PIL and the differences among PILs of individual patients were within $2 \%$.

Variations of the normal lung volumes receiving high to low doses of $30 \mathrm{~Gy}$ to $5 \mathrm{~Gy}$ (i.e., $V_{30 G y}$ to $V_{5 G y}$ ) are shown in Fig. 1. Largest lung sparing was observed in seven out of ten plans at $60 \%$ PIL, with up to $23.2 \%$ and $19.9 \%$ reduction in $V_{30 G y}$ to $V_{20 G y}(p<0.05)$, and at $70 \%$ PIL eight and nine out of ten plans produced better $V_{30 G y}$ and $V_{20 G y}(p<0.05)$ compared to the reference plans at $85 \%$ PIL, respectively. The absolute changes of the mean $V_{30 G y}$ and $V_{20 G y}$ were however negligible, amounting to $0.9 \%$ and $1.4 \%$ for $60 \%$ PIL plans, and $0.9 \%$ to $1.3 \%$ for $70 \%$ PIL plans, respectively. For the low dose lung volume $\left(\mathrm{V}_{10 G y}\right.$ and $\left.\mathrm{V}_{5 \mathrm{~Gy}}\right)$, three plans showed lowest $\mathrm{V}_{10 G y}$ and $V_{5 G y}$ at the $60 \%$ PIL while six and five plans showed lowest $V_{10 G y}$ and $V_{5 G y}$ at the $70 \%$ PIL, respectively. Figure 2 shows $V_{54 G y}$ and the mean dose to the normal lung included in the PTV (i.e., PTV minus GTV). Results of dose constraint parameters of other OARs were given in Table 1. Statistical tests showed significant differences between the reference $85 \%$ and other PILs mainly for dose metrics of target volumes and normal lung. The potential of PIL-VMAT to increase the tumor dose while keeping the critical organ doses was illustrated in Fig. 3.

Considering dose received by the chest wall and ribs, the $60 \%$ PIL resulted in the lowest averaged $V_{30 G y}$. However, the chest wall volume receiving dose $\geq 100 \%$ of the prescription dose is higher with the $60 \%$ PIL $(n=4)$ by 0.3 to $12.8 \mathrm{~cm}^{3}$ than with the $85 \%$ reference PIL in 4 lesions that abutted to the chest wall.

Both the target dose conformity (nCI) and the dose gradient $\left(R_{50 \%}\right)$ were found to be comparable between plans at different PILs $(p>0.05)$. Nonetheless, the $R_{50 \%}$ dose constraint criterion, suggested by Xiao et al. [22], was met by the reference $85 \%$ PIL in only one patient, by $80 \%$ PIL in two patients, by $70 \%$ PIL in four patients and by $60 \%$ PIL in five patients (Fig. 4). The 

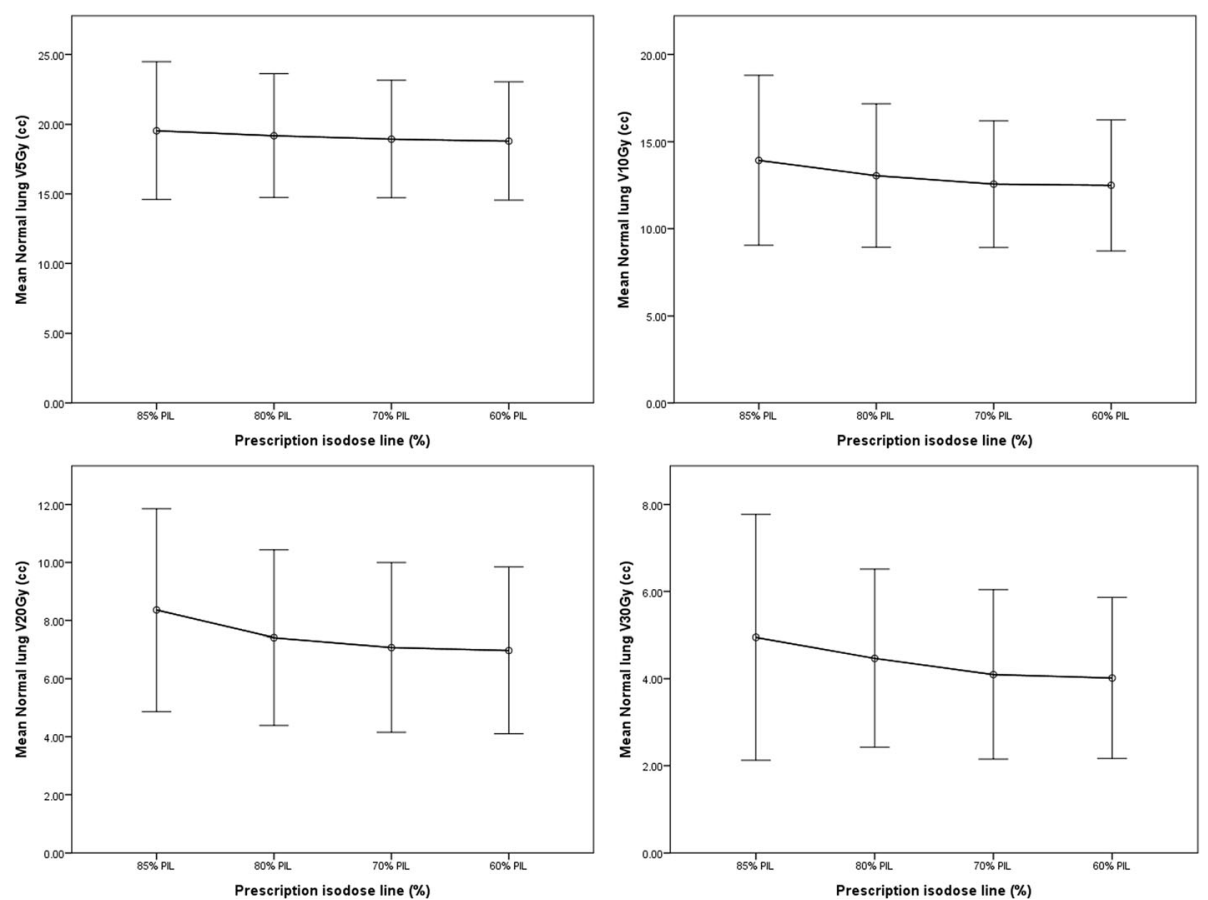

Fig. 1 Variations of averaged normal lung volumes receiving at least 5, 10, 20 and 30 Gy (i.e., $V_{5 G y,} V_{10 G y,} V_{20 G y,}$ and $V_{30 G y}$ ) as a function of prescription isodose levels. Error bars represent one standard deviations of $V_{5 G y}, V_{10 G y}, V_{20 G y}$ and $V_{30 G y}$

dose gradient was statistically better with $60 \%$ compared to the reference $85 \%$ PIL $(p<0.05)$.

\section{Correlation between PIL and LED}

Figure 5 shows the difference between the MC optimized and PB re-calculated plans in $D_{\text {mean }}$ and $D_{98 \%}$ of the PTV minus GTV border zone at varying PILs. The MC results predicted lower $D_{\text {mean }}$ and $D_{98 \%}$ than $\mathrm{PB}$, and the differences increased with decreasing PILs, indicating increasing magnitude of LED. It is noteworthy thought that significant dependence of PIL was observed only for $D_{\text {mean }}$ in the border zone.

\section{GTV-based prescription with varying PIL}

The effect of inverse-optimization with GTV-based prescription concepts was clearly demonstrated in Fig. 6. After re-normalizing the prescription such that $50 \%$ of the GTV received $54 \mathrm{~Gy}$, the peripheral dose of the PTV in terms of $D_{95 \%}$ remained reasonably high, ranging from 33.5 to $36.1 \mathrm{~Gy}$ at $60 \% \mathrm{IDL}$, and 38.3 to $39.8 \mathrm{~Gy}$ at $70 \%$ IDL, respectively. Normal tissue doses were further decreased with decreasing PIL (Table 2). When compared with the reference 85\% PIL plan employing PTV-based prescription, significant decreases of all normal tissue doses were achieved with $60 \%$ and $70 \%$ PIL by GTV-based prescription.
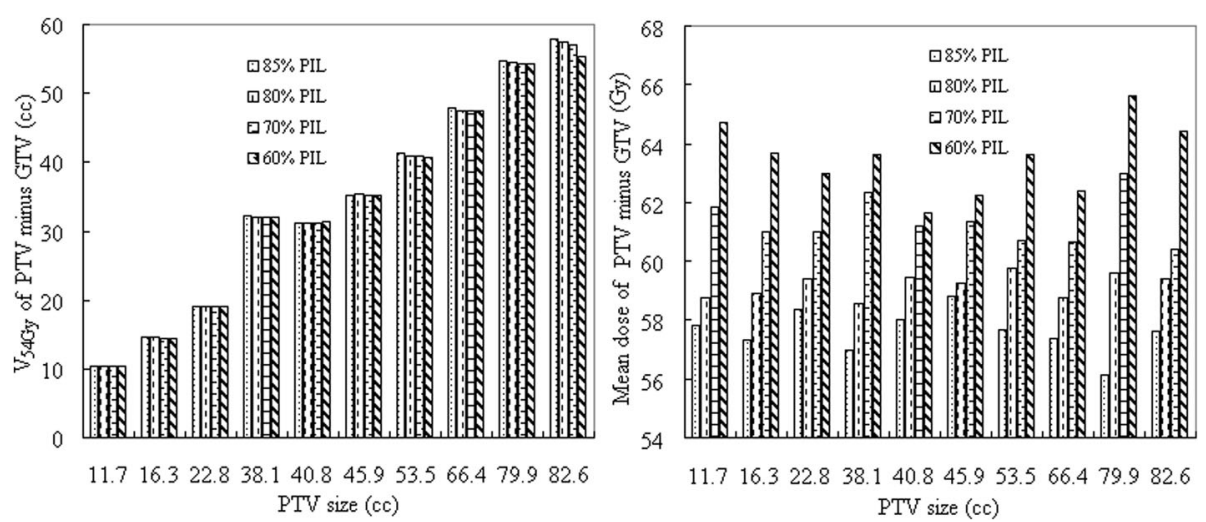

Fig. 2 (Left) shows the normal lung volume between the planning target volume (PTV) and the gross tumor volume (GTV) receiving at least 54 Gy $\left(V_{54 G y)}\right.$, and (right) the mean dose to this volume for individual lesions. Lesions are sorted according to size 
Table 1 Summary of the mean and one standard deviation of the dosimetric parameters obtained with PTV-based prescription

\begin{tabular}{|c|c|c|c|c|}
\hline & $85 \% \mathrm{PIL}$ & $80 \% \mathrm{PIL}$ & $70 \% \mathrm{PIL}$ & $60 \% \mathrm{PIL}$ \\
\hline$\overline{G T V} D_{\text {mean }}(G y)$ & $62.4 \pm 1.5$ & $66.2 \pm 1.0$ & $\mathrm{a} 74.4 \pm 1.1$ & $\mathrm{a} 84.2 \pm 2.3$ \\
\hline PTV D mean $(G y)$ & $58.7 \pm 0.9$ & $60.6 \pm 0.7$ & ${ }^{\mathrm{a}} 63.9 \pm 0.8$ & ${ }^{\mathrm{a}} 67.3 \pm 1.7$ \\
\hline PTV D $98 \%(G y)$ & $53.6 \pm 1.7$ & $53.1 \pm 0.2$ & ${ }^{a} 52.9 \pm 0.2$ & ${ }^{a} 52.6 \pm 0.4$ \\
\hline Spinal cord $D_{1 \%}(G y)$ & $9.1 \pm 2.9$ & $10.0 \pm 3.9$ & $9.7 \pm 3.8$ & $10.2 \pm 3.5$ \\
\hline Esophagus $D_{1 \%}$ (Gy) & $9.4 \pm 3.6$ & $9.6 \pm 4.9$ & $9.5 \pm 5.2$ & $9.6 \pm 5.2$ \\
\hline Bronchus $D_{1 \%}(\mathrm{~Gy})$ & $13.6 \pm 7.6$ & $13.2 \pm 7.6$ & $12.6 \pm 7.2$ & $12.0 \pm 7.2$ \\
\hline Trachea $D_{1 \%}(G y)$ & $5.3 \pm 7.1$ & $5.6 \pm 7.4$ & $5.1 \pm 6.9$ & $5.3 \pm 7.3$ \\
\hline Chest wall/Rib $V_{30 G y}(\mathrm{cc})$ & $42.0 \pm 35.7$ & $34.7 \pm 19.0$ & $33.1 \pm 22.1$ & $30.2 \pm 20.5$ \\
\hline Heart $D_{1 \%}(\mathrm{~Gy})$ & $10.7 \pm 9.4$ & $10.5 \pm 9.3$ & $10.0 \pm 9.0$ & $9.8 \pm 8.7$ \\
\hline PTV minus GTV D ${ }_{50 \%}(\mathrm{~Gy})$ & $57.6 \pm 0.7$ & $59.2 \pm 0.4$ & $61.4 \pm 0.8$ & $63.5 \pm 1.2$ \\
\hline PTV minus GTV $V_{54 G y}(\%)$ & $34.5 \pm 16.4$ & $34.3 \pm 16.2$ & $34.2 \pm 16.2$ & $34.0 \pm 15.9$ \\
\hline Normal Lung MLD (Gy) & $5.0 \pm 1.7$ & $4.7 \pm 1.4$ & $4.6 \pm 1.3$ & $4.6 \pm 1.4$ \\
\hline Normal Lung $V_{30 G y}(\%)$ & $4.9 \pm 2.8$ & $4.5 \pm 2.0$ & $4.1 \pm 1.9$ & ${ }^{\mathrm{a}} 4.0 \pm 1.8$ \\
\hline Normal Lung $V_{20 G y}(\%)$ & $8.4 \pm 3.5$ & $7.4 \pm 3.0$ & $7.1 \pm 2.9$ & ${ }^{a} 7.0 \pm 2.9$ \\
\hline Normal Lung $V_{\text {IOGy }}(\%)$ & $13.9 \pm 4.9$ & $13.0 \pm 4.1$ & ${ }^{a} 12.6 \pm 3.6$ & ${ }^{a} 12.5 \pm 3.8$ \\
\hline Normal Lung $V_{5 G y}(\%)$ & $19.5 \pm 4.9$ & $19.2 \pm 4.4$ & $18.9 \pm 4.2$ & $18.8 \pm 4.2$ \\
\hline $\mathrm{MU}$ & $4717 \pm 994$ & ${ }^{a} 4333 \pm 868$ & $4615 \pm 1013$ & $5011 \pm 1031$ \\
\hline $\mathrm{nCl}$ & $1.08 \pm 0.03$ & $1.07 \pm 0.02$ & $1.07 \pm 0.01$ & $1.07 \pm 0.01$ \\
\hline$R_{50 \%}$ & $6.11 \pm 1.42$ & $5.73 \pm 1.67$ & $5.11 \pm 1.26$ & ${ }^{a} 5.00 \pm 1.07$ \\
\hline
\end{tabular}

Abbreviations: GTV gross target volume, PTV planning target volume, $D_{\text {mean }}$ mean dose, $D_{x \%}$ dose to $x$ percent volume of the organ, $V_{x G y}$ percent volume of the organ receiving at least $x$ Gy, $M L D$ mean lung dose, $M U$ monitor unit, $n C l$ target dose conformity index, $R_{50 \%}$ dose gradient index, $P I L$ prescription isodose line

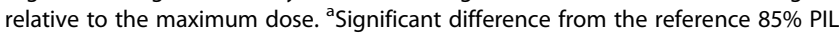

\section{Discussion}

This study demonstrated the technical feasibility of optimizing the PIL through its implicit relation with the lateral electronic disequilibrium (LED) to increase the dose gradient outside the target and hence improving the overall dosimetric quality in VMAT-based SBRT planning. This implicit relation was initially investigated in terms of the PTV dose differences between the PB and the $\mathrm{MC}$ dose calculation results for DCA-based lung SBRT [3]. This study followed the same methodology to understand the hypothesized LED origin of the dosimetric improvement. However, following the suggestion by Dish et al. [1] that the LED phenomenon can also be exploited in inverse optimization, our results were the first to show the dependence of PIL on LED for VMAT-type lung SBRT.

Using a reverse operation to re-calculate the type-B $\mathrm{MC}$ optimized dose plans with the type-A PB algorithms, as opposed to Zheng et al. [3] who re-calculated the $\mathrm{PB}$-optimized plans by the $\mathrm{MC}$ algorithms, we found significant increase of (negative) dose differences of mean dose in the low density lung tissue embedded in the PTV (i.e., PTV minus GTV) with decreasing PIL plans indicative of increased magnitude of LED at lower PIL. The net negative dose difference can be explained by the increased fluence using type-B algorithms at $60 \%$
PIL in striving to compensate the LED deficiency at the low density tissue dominant field edge. The increased fluence led to increased overall dose deposition that was assumed by the type-A PB algorithms. The $D_{2 \%}$ of the PTV border zone also showed similar trends of increasing (negative) dose differences with decreasing PIL despite insignificant statistical differences.

Unlike forward planning with DCA techniques, the advantage of inversely optimized VMAT is that the optimal MLC aperture relative to the tumor size can be solved through an intuitive adjustment of optimization parameters that are directly related to the clinical goals. Given the set of clinical goals, the optimizer would implicitly determine the optimal extent of LED adjusting for the photon beam energy as well as the variation of lung density between patients, avoiding the manual iteration of changing mostly the isotropic MLC margin to arrive at the desired PIL level, thus improving the planning efficiency. The direct incorporation of Monte Carlo dose engines further ensured that the dose distribution was robust against dosimetric errors caused by LED. Furthermore, this optimization approach is quite simple as we aimed at optimization of the PIL by adjusting the maximum and minimum dose (volume) to the target and the constraints of several dose controlling shell structures around the PTV. 



$85 \%$ PIL

$80 \%$ PIL

70\% PIL

$60 \%$ PIL

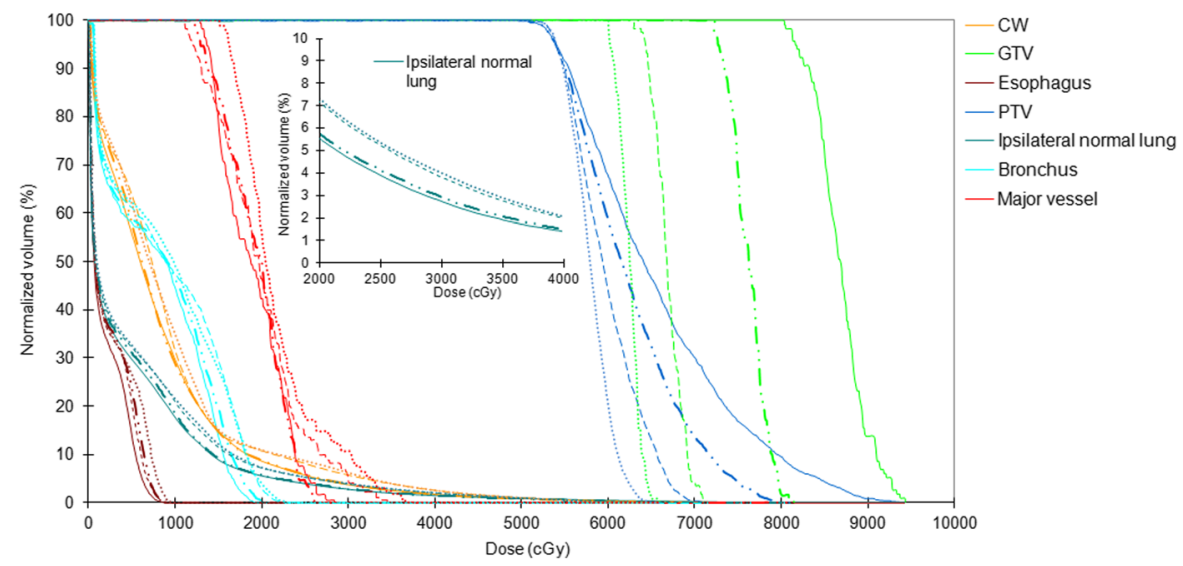

Fig. 3 Example dose distributions achieved with varying PIL-optimized VMAT plans in the axial plane and the dose-volume histograms (DVH) of selected organs. The PILs are displayed with 100\% corresponding to the treatment dose of 54 Gy. Inlet shows the magnified ipsilateral lung DVH



Fig. 4 The dose gradient index $R_{50 \%}$ for individual lesions sorted to size. Values of $R_{50 \%}$ suggested by Xiao et al. [22] are also given 

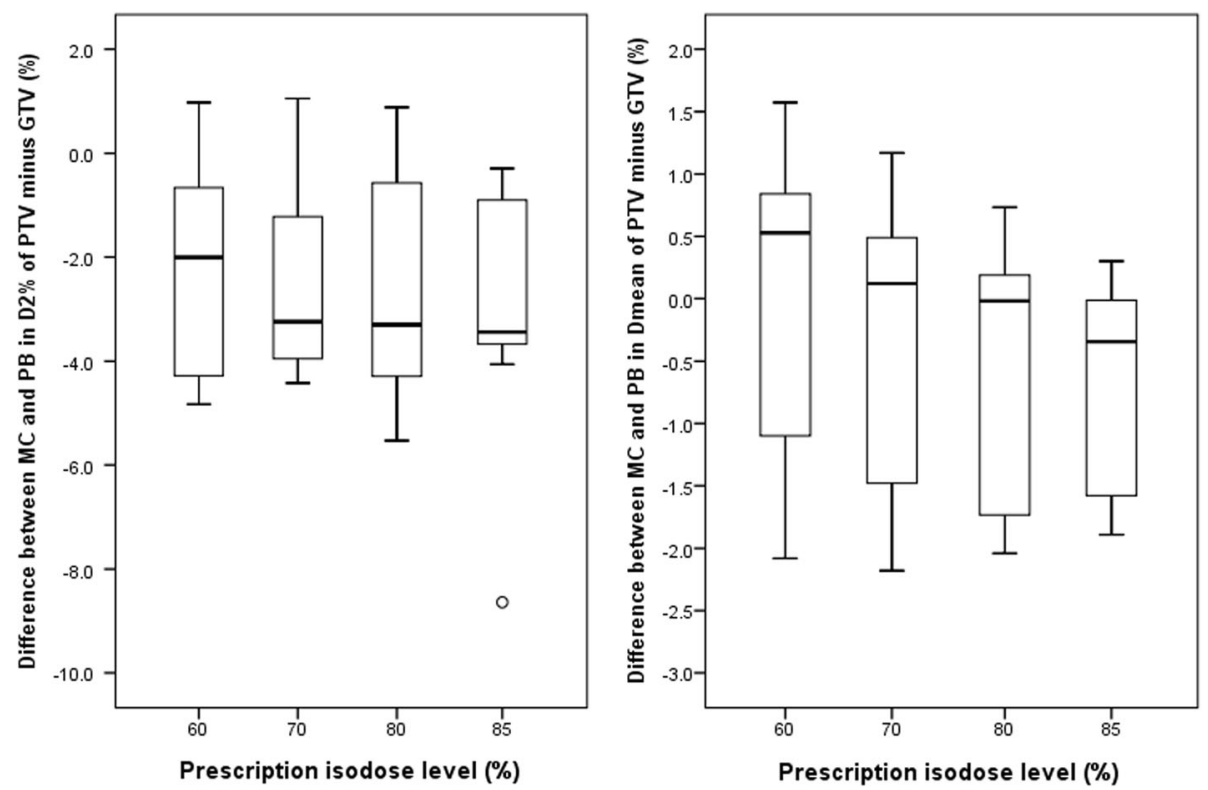

Fig. 5 Dependence of Monte Carlo (MC) and pencil beam (PB) dose differences to the mean dose $\left(D_{\text {mean }}\right)$ and dose to $2 \%\left(D_{2 \%}\right)$ of the lung tissue embedded in the planning target volume (PTV). Opened circles represent the individual patients and filled circles are the mean of all patients

Using this inverse optimization approach, the optimal PIL showing the most rapid dose falloff, which is significantly system design and planning technique dependent, was found to be between $60 \%$ and $70 \%$. These results were consistent with previous reports by Oku et al. [4] showing best plan at $60 \%$ PIL. Despite the theoretical benefits of lower PIL than $60 \%$, the associated toxicity profiles and local control remain largely unknown because it produced "hot spot" in the target beyond the acceptable range by most trial protocol [20, 21, 27] and therefore very limited clinical data are available. With the optimized PILs at $60 \%$ to $70 \%$ in present study, the GTV mean dose almost doubled in comparison to the reference 85\% PIL without increasing lung dose, although the dose between the PTV and GTV was on average higher at $70 \%$ and $60 \%$ PIL by $6 \%$ and $10 \%$, respectively. Considering this fact, it may be of genuine concern that the normal tissues embedded in this region, i.e. the non-tumorous margin region where motion and system inaccuracies are compensated for, receive higher doses. Given the treatment prescription of 54 Gy in 3 fractions delivered in 2 weeks for this study, $95 \%$ of the PTV will receive at least a biological effective dose (BED) of $151 \mathrm{~Gy}_{10}$, which is likely high enough to sterilize not just the tumor cells but also all other normal tissue cells as well. Yet, the increased chance of developing high grade radiation pneumonitis or fibrosis inside this small volume between GTV and PTV may be small as incidents of radiation pneumonitis or fibrosis are generally correlated with mean lung dose or low dose lung volume which were not

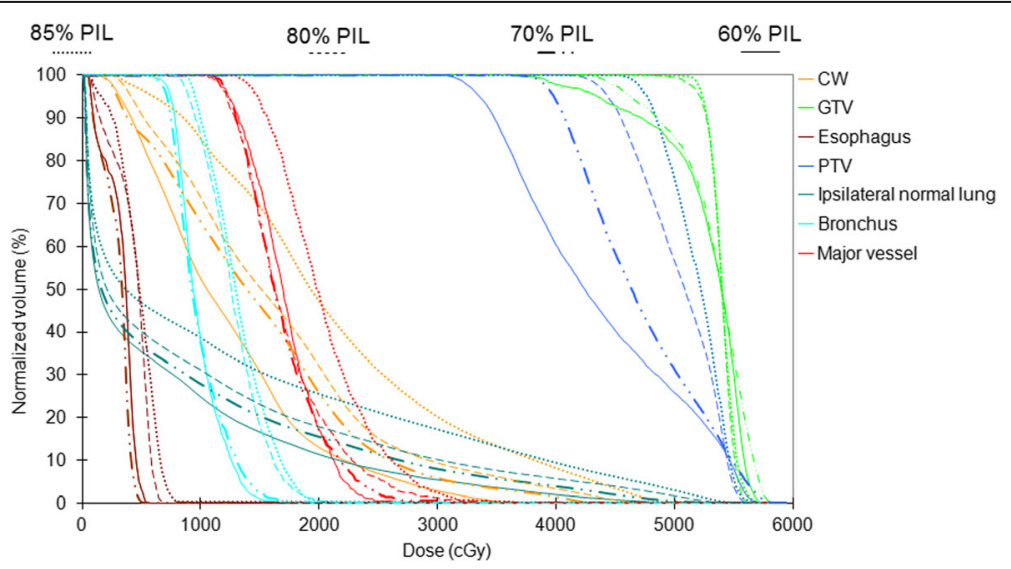

Fig. 6 Dose-volume histograms of the same patient in Fig. 4, resulted from re-prescribing to the GTV mean dose, i.e. GTV-based prescription 
Table 2 Summary of the mean and one standard deviation of the dosimetric parameters obtained with GTV-based prescription

\begin{tabular}{|c|c|c|c|c|}
\hline & $85 \%$ PIL & $80 \%$ PIL & $70 \%$ PIL & $60 \% \mathrm{PIL}$ \\
\hline GTV D mean $(G y)$ & $54.0 \pm 0.1$ & $53.9 \pm 0.1$ & $53.6 \pm 0.2$ & $54.0 \pm 1.2$ \\
\hline PTV D mean $(G y)$ & $50.7 \pm 0.6$ & $49.3 \pm 0.7$ & $46.1 \pm 0.9$ & $42.9 \pm 1.1$ \\
\hline PTV $D_{98 \%}(G y)$ & $45.9 \pm 1.2$ & $43.2 \pm 0.8$ & $38.2 \pm 0.5$ & $33.6 \pm 1.1$ \\
\hline PTV D $95 \%$ (Gy) & $46.7 \pm 1.1$ & $43.9 \pm 0.7$ & $39.0 \pm 0.4$ & $34.4 \pm 0.9$ \\
\hline Spinal cord $D_{1 \%}(\mathrm{~Gy})$ & $7.9 \pm 2.6$ & $8.1 \pm 3.2$ & $7.0 \pm 2.7$ & ${ }^{\mathrm{a}} 6.5 \pm 2.2$ \\
\hline Esophagus $D_{1 \%}(\mathrm{~Gy})$ & $8.2 \pm 3.0$ & $7.8 \pm 4.1$ & ${ }^{\mathrm{a}} 6.9 \pm 3.8$ & ${ }^{\mathrm{a}} 6.1 \pm 3.3$ \\
\hline Bronchus $D_{1 \%}(\mathrm{~Gy})$ & $11.8 \pm 6.6$ & $10.9 \pm 6.2$ & ${ }^{\mathrm{a}} 9.1 \pm 5.2$ & ${ }^{a} 7.6 \pm 4.5$ \\
\hline Trachea $D_{1 \%}(G y)$ & $4.6 \pm 6.1$ & $4.5 \pm 6.1$ & ${ }^{a} 3.7 \pm 5.0$ & $\mathrm{a}_{3.4 \pm 4.6}$ \\
\hline Chest wall/Rib V з 0 Gy $_{\text {(cc) }}$ & $29.8 \pm 27.3$ & $18.1 \pm 16.3$ & ${ }^{\mathrm{a}} 12.4 \pm 12.4$ & ${ }^{\mathrm{a}} 6.3 \pm 8.0$ \\
\hline Heart $D_{1 \%}(\mathrm{~Gy})$ & $9.2 \pm 8.3$ & $8.5 \pm 7.5$ & $7.2 \pm 6.5$ & ${ }^{\mathrm{a}} 6.3 \pm 5.5$ \\
\hline PTV minus GTV $D_{50 \%}(G y)$ & $49.8 \pm 0.8$ & $48.2 \pm 0.6$ & ${ }^{\mathrm{a}} 44.3 \pm 0.6$ & ${ }^{a} 40.5 \pm 0.6$ \\
\hline PTV minus GTV $V_{54 G y}(\%)$ & $1.0 \pm 1.8$ & $1.3 \pm 1.5$ & ${ }^{\mathrm{a}} 0.4 \pm 0.5$ & ${ }^{\mathrm{a}} 0.1 \pm 0.1$ \\
\hline Normal Lung MLD (Gy) & $4.4 \pm 1.6$ & $4.0 \pm 1.4$ & ${ }^{a} 3.3 \pm 1.0$ & ${ }^{a} 2.9 \pm 0.9$ \\
\hline Normal Lung $V_{30 G y}(\%)$ & $4.0 \pm 2.6$ & $3.1 \pm 1.6$ & ${ }^{\mathrm{a}} 2.2 \pm 1.2$ & ${ }^{\mathrm{a}} 1.7 \pm 0.9$ \\
\hline Normal Lung $V_{20 G y}(\%)$ & $7.0 \pm 3.6$ & $5.9 \pm 2.6$ & ${ }^{a} 4.7 \pm 2.2$ & ${ }^{a} 3.7 \pm 1.7$ \\
\hline Normal Lung $V_{\text {IOGy }}(\%)$ & $12.8 \pm 4.9$ & $11.3 \pm 3.9$ & ${ }^{\mathrm{a}} 9.8 \pm 3.5$ & ${ }^{\mathrm{a}} 9.4 \pm 4.5$ \\
\hline Normal Lung $V_{5 G y}(\%)$ & $18.2 \pm 5.0$ & $17.2 \pm 4.2$ & ${ }^{\mathrm{a}} 15.6 \pm 3.7$ & ${ }^{a} 14.6 \pm 3.8$ \\
\hline
\end{tabular}

PIL prescription isodose line relative to the maximum dose, GTV gross target volume, $P T V$ planning target volume, Dmean mean dose, Dx dose to $x$ percent volume of the organ, $V x$ percent volume of the organ receiving at least $x \mathrm{~Gy}, M U$ monitor unit, $n C l$ target dose conformity index, $R_{50 \%}$ dose gradient index. ${ }^{\text {aS }}$ ignificant difference from the reference $85 \%$ PIL based on PTV $D_{95 \%}$ prescription

increased at the lower 70-60\% PIL [28]. Such assumption also has support from the recent DEGRO guidelines published by the German SBRT working group for early stage NSCLC which recommended a maximum dose of $150 \%$ to the PTV (i.e., 65\% PIL) based on the clinical evidences from a large-scale multi-center study [9].

Furthermore, this study clearly demonstrated that besides lung doses at optimized PIL as low as $60 \%$ doses to other serial OARs such as esophagus, heart, bronchus and trachea, major vessels, and spinal cord did not increase even for central tumors because the optimizer would automatically determine the set of anisotropic MLC margins variable with the gantry angle to achieve the specified dose constraints. This is generally impractical and labor-intensive in forward planning with DCA techniques. One of the exceptions could be tumors having the PTV overlapped with the chest walls. Nevertheless, this problem can be partly addressed by imposing more stringent dose-volume constraints on the chest wall and rib structures to push away the high dose. Also, the plan quality metric of the target dose conformity showed no statistical differences between different PILs. The dose gradient index was better for plans with $60 \%$ PIL than 80 and $85 \%$ PIL $(p<0.05)$ and was comparable between 60 and 70\% PIL plans. The monitor units were found to increase with decreasing PIL in a linear manner likely resulting in minimally increased treatment time.

The additional advantage that comes along with optimized PIL at $\sim 60$ to $70 \%$ is the potential of further margin reduction. In this study, the mid-ventilation PTV was based on the van Herk's margin recipe and calculated assuming a reference $85 \%$ PIL. The theoretical margin may be decreased by 1 to $2 \mathrm{~mm}$ from 85 to $60 \%$ PIL for our patient cohort whose observed motion was up to $2 \mathrm{~cm}$, mainly due to the smaller $\beta$ value of the inverse cumulative standard normal distributions at the prescribed PTV minimum dose level [19]. This margin reduction, although small, may leverage the dosimetric benefits of inverse optimization in VMAT even further, though we acknowledge that further studies are required in this regard and a discussion if the van Herk's margin recipe can be used for inhomogeneous dose distributions is beyond the scope of this work.

Clinically, a BED of $100 \mathrm{~Gy}_{10}$, assuming an $\alpha / \beta$ ratio of $10 \mathrm{~Gy}$, has been universally recognized as the approximate threshold dose to achieve adequate local control in early stage NSCLC, however, only based on a prescription to the PTV periphery [7, 29, 30] and with unclear PIL. On the other hand, Guckenberger et al. [7] recently hinted at lower PIL (<80\%) being significantly superior in local control in their data $(86.8 \%$ vs. $69.1 \%, p=0.005)$. Recently, the same retrospective large-scale multi-center study have reported on local control being also significantly dependent of the maximum isocenter dose $[9,10]$ which strongly supports our investigation of decreasing the PIL through inverse VMAT optimization and with that increasing the GTV doses. Following that concept and going one step further, a PTV prescription 
dose of $3 \times 18$ Gy at $85 \%$ PIL (151 Gy G $_{10}$ to PTV D $95 \%$ and $D_{\text {max }}=198 \mathrm{~Gy}_{10}$, reference dose level in our study) may very likely be reduced to $3 \times 14$ Gy at $60 \%$ PIL (101 Gy $y_{10}$ to PTV $D_{95 \%}$ and $D_{\max }=233 \mathrm{~Gy}_{10}$ ), resulting in substantial reductions in dose to OARs and even possibly in an increase in tumor control probability (TCP).

Based on this idea, this study further investigated the potential of significant dose de-escalation for SBRT by making best use of the physics derivable from the LED phenomenon. When we prescribed the dose in a way that GTV $D_{50 \%}$ equals 54 Gy $\left(B E D=151 \mathrm{~Gy}_{10}\right)$ for all PIL with the aim to keep the TCP constant, the resulting BED in the PTV $D_{95 \%}$ ranged from 71.0 to $79.5 \mathrm{~Gy}_{10}$, and 87.1 to $92.7 \mathrm{~Gy}_{10}$ at $60 \%$ and $70 \%$ PIL, respectively. While such PTV doses may seem low in comparison to previous publications, a recent investigation of PTV prescription dose reduction with constant high GTV mean doses found high local control for lung tumors even with low PTV prescription doses (PTV D $95 \%$ BED $\geq 89.7 \mathrm{~Gy}_{10}$ vs. $<89.7 \mathrm{~Gy}_{10}$, hazard ratio 0.077, confidence interval $0.012-0.503, p=0.001$,) [13]. Hence, we may hypothesize that $3 \times 18$ Gy prescribed to the mean GTV dose at 60 to $70 \%$ PIL may still result in high LC (> 90\% [13]) while substantially reduce the dose to the lungs and other OARs.

In practice, prescription based on the GTV mean dose can be optimized for different combinations of dose fractionation schedule and PIL. For example, 54 Gy GTV mean dose could be delivered in 4 fractions at 60\% PIL, producing PTV $D_{95 \%}$ of $79.5 \mathrm{~Gy}_{10}$ that is roughly equivalent to $48 \mathrm{~Gy}$ in 4 fractions prescribed to the isocenter as commonly practiced in Japan [27]. For dose fractionation schedules with more fractions, 54 Gy GTV mean dose could be delivered in 5 fractions, but at slightly higher PIL at $~ 67 \%$, producing PTV $D_{95 \%}$ of $66.2 \mathrm{~Gy}_{10}$ equivalent to 50 Gy in 5 fractions reported by Aoki et al. [11] Alternatively, the prescription can also be re-normalized to higher median GTV dose than 54 Gy to achieve the desired PTV $D_{95 \%}$ covered by the optimal PIL. Table 3 is provided to predict the 3-year local control rate from different dose schedules and prescription methods reported in the literature and in this study.

Nevertheless, it is worthwhile to note that the concept of GTV-based prescription for lung SBRT and outcomes for such method are exclusively limited to robotic SBRT so far $[13,15]$, although the data is part of and fits nicely to recent TCP modeling $[9,10]$. Clinical implementation of the proposed dose de-escalation approach by optimized PIL at $\sim 60 \%$ with other techniques such as VMAT must be taken with great cautions, and further clinical studies are warranted to validate its efficacy and safety. It is admitted that this study did not fully address the implementation issues of GTV-based prescription. Future studies would be required to develop a link of the GTV prescription with the conventional concept of PTV prescription isodose line, the dose encompassing level of the PTV and their interactions with other patient-dependent / treatment technique-specific factors such as tumor motion range, PTV definition, etc. Further limitations to this study come from the limited number of presented cases as other parameter such as lesion location, volume and dimensions or density of the lesions itself could not have been statistically investigated.

\section{Conclusions}

Optimizing the Monte Carlo calculated prescription isodose level for VMAT has the obvious advantage of improving the dosimetric quality of lung SBRT treatment plans and offers the possibility to achieve dose de-escalation. Further clinical investigation of gross tumor volume based dose prescription and optimal prescription isodose levels are warranted.

Table 3 Summary of the biological effective dose (BED) to 95\% of the planning target volume (PTV D95\%) and the 3-year local control (LC) rates in the literature

\begin{tabular}{|c|c|c|c|c|c|c|c|c|}
\hline & Prescription & $\begin{array}{l}\text { Isodose level } \\
\text { at PTV edge }\end{array}$ & Dose algorithm & Total dose & $\begin{array}{l}\text { BED }\left(G y_{10}\right) \text { in } \\
3 \text { fractions }\end{array}$ & $\begin{array}{l}\text { BED }\left(G y_{10}\right) \text { in } \\
4 \text { fractions }\end{array}$ & $\begin{array}{l}\text { BED }\left(G_{10}\right) \text { in } \\
5 \text { fractions }\end{array}$ & 3-years LC \\
\hline Aoki et al. [11] & Isocenter & $80 \%$ & Type-A & 50 & & & 66.2 & $95.0 \%$ \\
\hline Onishi et al. [12] & & & & & & & & $95.0 \%$ \\
\hline Yoshitaki et al. [31] & Isocenter & $80 \%$ & Type-A & 48 & & 62.3 & & $90.0 \%$ \\
\hline Shibamoto et al. [32] & & & & & & & & $87.0 \%$ \\
\hline Ricardi et al. [33] & PTV edge & $80 \%$ & Type-A & 45 & 99.0 & & & $87.8 \%$ \\
\hline Hassbeek et al. [34] & PTV edge & $80 \%$ & Type-A & $\begin{array}{l}60 \\
60\end{array}$ & 145.7 & & 108.6 & $\begin{array}{l}89.3 \% \\
89.3 \%\end{array}$ \\
\hline \multirow[t]{2}{*}{ This study } & GTV mean dose & $70 \%$ & Type-B & 54 & 89.2 & 76.7 & 69.1 & \\
\hline & & $60 \%$ & Type-B & & 74.3 & 64.4 & 58.4 & \\
\hline
\end{tabular}

Note: $\mathrm{BED}_{10}(a / \beta=10 \mathrm{~Gy})$ was calculated after adjusting the physical prescription dose by a factor of 0.88 for type-A algorithm with respect to type-B Monte Carlo algorithm according to Ref [37] 


\section{Abbreviations}

3DCT: Three-dimensional computed tomography; 4DCT: Four-dimensional computed tomography; BED: Biological effective dose; DVH: Dose-volumehistogram; GTV: Gross target volume; LED: Lateral electronic disequilibrium; MC: Monte Carlo; MidV: Mid-ventilation; MLC: Multi-leaf collimator; MLD: Mean lung dose; MU: Monitor units; NSCLC: Non-small cell lung carcinoma; OAR: Organ-at-risks; PB: Pencil beam; PIL: Prescription isodose line; PTV: Planning target volume; SBRT: Stereotactic body radiotherapy; TCP: Tumor control probability; VMAT: Volumetric-modulated arc radiotherapy

\section{Acknowledgements}

N/A

\section{Funding}

The study did not receive funding.

Availability of data and materials

The data will be made available upon request.

\section{Authors' contributions}

MC designed the study and prepared the manuscript. MW, RL, SC and MC generated the study treatment plans. RL, $M C$ and $O B$ analyzed the results. $M C$ and $O B$ designed and offered the final proof-read of the data and manuscript. All authors read and approved the final manuscript.

\section{Ethics approval and consent to participate}

The study was approved by the New Territories West Cluster Clinical and Research Ethics Committee (reference number: NTWC/CREC/15138) and ethics approval was not required.

\section{Consent for publication}

N/A

\section{Competing interests}

The authors declare that they have no competing interest.

\section{Publisher's Note}

Springer Nature remains neutral with regard to jurisdictional claims in published maps and institutional affiliations.

\section{Author details}

'University Medical Center Schleswig-Holstein, Campus Kiel, Department for Radiation Oncology, Arnold-Heller-Straße 3, Haus 50, Karl-LennertKrebscentrum Nord, 24105 Kiel, Germany. ${ }^{2}$ Imperial College London Healthcare NHS Trust, Department of Radiation Physics, London, UK. ${ }^{3}$ Tuen Mun Hospital, Department of Clinical Oncology, Special Administrative Region of China, Hong Kong, Hong Kong, Special Administrative Region of China. ${ }^{4}$ Saphir Radiosurgery Center Northern Germany, Güstrow, Germany.

Received: 20 March 2017 Accepted: 29 January 2018

Published online: 09 February 2018

\section{References}

1. Disher B, Hajdok G, Gaede S, Mulligan M, Battista J. Forcing lateral electron disequilibrium to spare lung tissue: a novel technique for stereotactic body radiation therapy of lung cancer. Phys Med Biol. 2013;58:6641.

2. Ojala J, Kapanen M. Quantification of dose differences between two versions of Acuros XB algorithm compared to Monte Carlo simulations - the effect on clinical patient treatment planning. J Appl Clin Med Phys. 2015;16:213-25.

3. Zheng D, Zhang Q, Liang X, Zhu X, Verma V, Wang S, Zhou S. Effect of the normalized prescription isodose line on the magnitude of Monte Carlo vs. pencil beam target dose differences for lung stereotactic body radiotherapy. J Appl Clin Med Phys. 2016;17:48-58.

4. Oku Y, Takeda A, Kunieda E, Sudo Y, Oooka Y, Aoki Y, Shimouchi Y, Nishina R, Nomura K, Sugiura M, Ohashi T. Analysis of suitable prescribed isodose line fitting to planning target volume in stereotactic body radiotherapy using dynamic conformal multiple arc therapy. Pract Radiat Oncol. 2012;2:46-53.
5. Ding C, Solberg TD, Hrycushko B, Xing L, Heinzerling J, Timmerman RD. Optimization of normalized prescription isodose selection for stereotactic body radiation therapy: conventional vs robotic linac. Med Phys. 2013;40(5):051705.

6. Takeda A, Oku Y, Sanuki N, Eriguchi T, Aoki Y, Enomoto T, Kaneko T, Nishimura S, Kunieda E. Feasibility study of stereotactic body radiotherapy for peripheral lung tumors with a maximum dose of $100 \mathrm{~Gy}$ in five fractions and a heterogeneous dose distribution in the planning target volume. J Radiat Res. 2014:55:988-95.

7. Guckenberger M, Allgäuer M, Appold S, Dieckmann K, Ernst I, Ganswindt U, Holy R, Nestle U, Nevinny-Stickel M, Semrau S, et al. Safety and efficacy of stereotactic body radiotherapy for stage 1 non-small-cell lung cancer in routine clinical practice: a patterns-of-care and outcome analysis. J Thorac Oncol. 2013:8:1050-8.

8. Bibault J-E, Mirabel X, Lacornerie T, Tresch E, Reynaert N, Lartigau E. Adapted prescription dose for Monte Carlo algorithm in lung SBRT: clinical outcome on 205 patients. PLoS One. 2015;10:e0133617.

9. Guckenberger M, Klement RJ, Allgäuer M, Andratschke N, Blanck O, Boda-Heggemann J, Dieckmann K, Duma M, Ernst I, Ganswindt U, et al. Local tumor control probability modeling of primary and secondary lung tumors in stereotactic body radiotherapy. Radiother Oncol. 2016;118:485-91.

10. Klement RJ, Allgäuer M, Andratschke N, Blanck O, Boda-Heggemann J, Dieckmann K, Duma M, Ernst I, Flentje M, Ganswindt U, et al. Bayesian cure rate modeling of local tumor control: evaluation in stereotactic body radiation therapy for pulmonary metastases. Int J Radiat Oncol Biol Phys. 2016;94:841-9.

11. Aoki M, Hatayama Y, Kawaguchi H, Hirose K, Sato M, Akimoto H, Fujioka I, Ono S, Tsushima E, Takai Y. Clinical outcome of stereotactic body radiotherapy for primary and oligometastatic lung tumors: a single institutional study with almost uniform dose with different five treatment schedules. Radiat Oncol. 2016;11:5.

12. Onishi H, Araki T. Stereotactic body radiation therapy for stage I nonsmall-cell lung cancer: a historical overview of clinical studies. Jpn J Clin Onco. 2013;43:345-50.

13. Stera S, Balermpas $P$, Chan MKH, Huttenlocher $S$, Wurster $S$, Keller C, Imhoff D, Rades D, Dunst J, Rödel C, et al. Breathing-motion-compensated robotic guided stereotactic body radiation therapy. Strahlenther Onkol. 2018;194(2): 143-55.

14. Seuntjens J, Lartigau EF, Cora S, et al. ICRU Report 91. J ICRU. 2014;14(2):NP.

15. Lacornerie T, Lisbona A, Mirabel X, Lartigau E, Reynaert N. GTV-based prescription in SBRT for lung lesions using advanced dose calculation algorithms. Radiat Oncol. 2014;9:223.

16. Miura H, Masai N, Oh R-J, Shiomi H, Yamada K, Sasaki J, Usmani M, Inoue T. Dosimetric comparison of different prescription modes in lung stereotactic body radiation therapy. International Journal of Medical Physics, Clinical Engineering and Radiation Oncology. 2014:14-9.

17. Miura H, Masai N, Oh R-J, Shiomi H, Sasaki J, Inoue T. Approach to dose definition to the gross tumor volume for lung cancer with respiratory tumor motion. J Radiat Res. 2013;54:140-5.

18. Chan MKH, Kwong DLW, Law GML, Tam E, Tong A, Lee V, Ng SCY. Dosimetric evaluation of four-dimensional dose distributions of CyberKnife and volumetric-modulated arc radiotherapy in stereotactic body lung radiotherapy. J Appl Clin Med Phys. 2013;14:136-49.

19. Sonke J-J, Rossi M, Wolthaus J, van Herk M, Damen E, Belderbos J. Frameless stereotactic body radiotherapy for lung cancer using four-dimensional cone beam CT guidance. Int J Radiat Oncol Biol Phys. 2009;74:567-74.

20. Hurkmans CW, Cuijpers JP, Lagerwaard FJ, Widder J, van der Heide UA, Schuring D, Senan S. Recommendations for implementing stereotactic radiotherapy in peripheral stage IA non-small cell lung cancer: report from the quality assurance working party of the randomised phase III ROSEL study. Radiat Oncol. 2009:4:1.

21. Stanic S, Paulus R, Timmerman RD, Michalski JM, Barriger RB, Bezjak A, Videtic GM, Bradley J. No clinically significant changes in pulmonary function following stereotactic body radiation therapy for early- stage peripheral non-small cell lung cancer: an analysis of RTOG 0236. Int J Radiat Oncol Biol Phys. 2014;88(5):1092-9.

22. Xiao Y, Papiez L, Paulus R, Timmerman R, Straube WL, Bosch WR, Michalski J, Galvin JM. Dosimetric evaluation of heterogeneity corrections for RTOG 0236: stereotactic body radiotherapy of inoperable stage I-II non-small-cell lung cancer. Int J Radiat Oncol Biol Phys. 2009;73:1235-42.

23. Videtic GM, Hu C, Singh AK, Chang J4, Parker W, Olivier KR, Schild SE, Komaki R, Urbanic JJ, Timmerman RD, Choy H. A randomized phase 2 study 
comparing 2 stereotactic body radiation therapy schedules for medically inoperable patients with stage I peripheral non-small cell lung cancer: NRG Oncology RTOG 0915 (NCCTG N0927). Int J Radiat Oncol Biol Phys. 2015; 93(4):757-64

24. Fippel M. Fast Monte Carlo dose calculation for photon beams based on the VMC electron algorithm. Med Phys. 1999;26:1466-75.

25. Paddick I. A simple scoring ratio to index the conformity of radiosurgical treatment plans technical note. J Neurosurg. 2000;93:219-22.

26. Jeleń U, Alber M. A finite size pencil beam algorithm for IMRT dose optimization: density corrections. Phys Med Biol. 2007;52:617.

27. Nagata Y, Hiraoka M, Shibata T, Onishi H, Kokubo M, Karasawa K, Shioyama Y, Onimaru R, Kunieda E, Ishikura S. A phase II trial of stereotactic body radiation therapy for operable T1NOMO non-small cell lung cancer: Japan clinical oncology group (JCOG0403). Int J Radiat Oncol Biol Phys. 2010;78:S27-8.

28. Barriger RB, Forquer JA, Brabham JG, Andolino DL, Shapiro RH, Henderson MA, Johnstone PAS, Fakiris AJ. A dose-volume analysis of radiation pneumonitis in non-small cell lung cancer patients treated with stereotactic body radiation therapy. Int J Radiat Oncol Biol Phys. 2012;82:457-62.

29. Chang JY, Li Q-Q, Xu Q-Y, Allen PK, Rebueno N, Gomez DR, Balter P, Komaki R, Mehran R, Swisher SG, Roth JA. Stereotactic ablative radiation therapy for centrally located early stage or isolated parenchymal recurrences of nonsmall cell lung cancer: how to fly in a "no fly zone"? Int J Radiat Oncol Biol Phys. 2014;88:1120-8.

30. Grills IS, Hope AJ, Guckenberger M, Kestin LL, Werner-Wasik M, Yan D, Sonke J-J, Bissonnette J-P, Wilbert J, Xiao Y, Belderbos J. A collaborative analysis of stereotactic lung radiotherapy outcomes for early-stage non-small-cell lung cancer using daily online cone-beam computed tomography image-guided radiotherapy. J Thorac Oncol. 2012;7:1382-93.

31. Yoshitake T, Nakamura K, Shioyama Y, Sasaki T, Ohga S, Shinoto M, Terashima K, Asai K, Matsumoto K, Matsuo Y, Baba S, Honda H. Stereotactic body radiation therapy for primary lung cancers clinically diagnosed without pathological confirmation: a single-institution experience. Int J Clin Oncol. 2015;20(1):53-8.

32. Shibamoto Y, Hashizume C, Baba F, Ayakawa S, Miyakawa A, Murai T, Takaoka T, Hattori Y, Asai R. Stereotactic body radiotherapy using a radiobiology-based regimen for stage I non-small-cell lung cancer: five-year mature results. J Thorac Oncol. 2015;10(6):960-4.

33. Ricardi U, Filippi AR, Guarneri A, Giglioli FR, Ciammella P, Franco P, Mantovani C, Borasio P, Scagliotti GV, Ragona R. Stereotactic body radiation therapy for early stage non-small cell lung cancer: Results of a prospective trial. Lung Cancer. 2010;68(1):72-77.

34. Haasbeek CJ, Lagerwaard FJ, Cuijpers JP, Slotman BJ, Senan S. Is adaptive treatment planning required for stereotactic radiotherapy of stage I non-small-cell lung cancer? Int J Radiat Oncol Biol Phys. 2007;67(5):1370-4.

\section{Submit your next manuscript to BioMed Central and we will help you at every step:}

- We accept pre-submission inquiries

- Our selector tool helps you to find the most relevant journal

- We provide round the clock customer support

- Convenient online submission

- Thorough peer review

- Inclusion in PubMed and all major indexing services

- Maximum visibility for your research

Submit your manuscript at www.biomedcentral.com/submit

) Biomed Central 